\title{
Comparison between two different quantum models of ball lightning
}

\author{
Vladimir Kuznetsov ${ }^{1, *}$ \\ ${ }^{1}$ Institute of Cosmophysical Research and Radio Wave Propagation FEB RAS, Paratunka, Kamchatka, \\ Russia
}

\begin{abstract}
Two models of ball lightning are compared here. Each model is quantum. One of them was proposed by American and Finnish physics [1], who were the first to create three-dimensional skyrmions - particles in BoseEinstein condensate with an ordered spin structure where central and boundary spins are opposite directed. A stable knot between electric and magnetic fields in a three-dimensional skyrmion is treated by the authors as a quantum model of ball lightning (BL). The next model proposed here proceeds from quantum entanglement (QE) of protons in hydrogen bonds (HBP) inside atmosphere oversaturated water vapour [2]. Two other quantum models issued in 1937 and 2007 are mentioned here. Origin of high-energy particles flux inside clouds as conditions for its origin when dark lightning [3] precede ordinary one creating additional ionization, fluxes of fast electrons with $\mathrm{MeV}$ energy prior to lightning which is initiating BL, glow, sprites are considered. All phenomena listed above appear to be of general nature resulting from QE of HBP in overstable water clusters (tetramers) inside clouds. Comparing models introduced above we use the estimation of BL capacity to enter via glass as a key parameter. Unexplained in BL models this frequently observed phenomenon as well as many other such as the BL transparency, its coldness and hiss become obviously explicable in the context of our model.
\end{abstract}

\section{Introduction}

The problem of BL interested me as many other physicists in Russia in the mid-60s, when I worked at the Novosibirsk Institute of nuclear physics (INP) and was engaged in gas discharge physics. What can we say, if the Nobel laureate, academician P. L. Kapitsa published an article about the model of BL (ref. in [2]). With physics students he went to the Caucasus where he tried to register the radio emission of BL, as it followed from his model (1955). In 1964 the newspaper "Komsomolskaya Pravda" published an article "Storeroom of lightning" which interested many young people. At that time the article of Dmitriev about his observing of BL on the Oka river (1967) appeared.

We, as the young employees at INP, discussed the information on BL available to us, we tried to create models, conducted experiments on gas discharge. We got spherical discharges, but they were not BL. The most apparent cluster model of BL was developed by I.P.Stakhanov (1985). It is worth noting the work of Smirnov B. M. (1990).

\footnotetext{
*e-mail: vvkuz38@mail.ru

This work was supported by project NoAAAA-A17-117080110043-4
} 
In the zero years when fundamentally new ideas on quantum nonlocality (cohesion, entanglement, etc.) appeared I returned to the problem of BL and tried to find its solution within the framework of many-particle quantum entanglement [2]. The problem about BL penetration through the smallest hole in the glass remained unsolved. I got acquainted with a large number of articles on BL models and its behavior in nature and in the laboratory. None of these models explain this fact. It should be remarked that the authors of these models did not pay attention to it, from which it followed that the survivability and stability of BL should be provided by its elements with a very small size with a micron order, i.e., otherwise, at the quantum level. Fig. 1 shows how BL penetrates through the smallest hole.

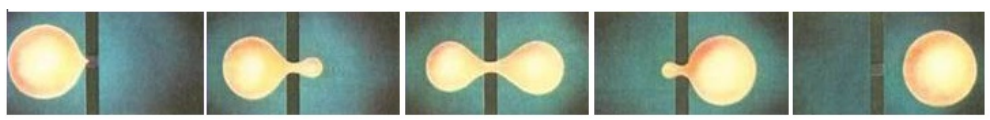

Figure 1. BL penetrates through the smallest hole.

The situation changed after I got interested in the problem of dark lightning (DL). Discovered as a phenomenon in 1991 [3] DL is a flux of fast electrons with speeds close to light one, that sharply distinguishes it from ordinary lightning whose electrons are not so rapid. Colliding with air molecules, the ultrafast electrons of DL (called "escaping" electrons) generate gamma rays, which produce electrons and their antiparticles, whose subsequent annihilation with air generate even more gamma rays. We can assume that the DL is an additional air ionizer, which helps the lightning to break through the gas gap. There were proposals to use galactic cosmic rays as an additional ionizer (references in [3]).

Until recently, it was believed that DL occurs no more than a thousand or several thousand times a year. The authors (ref. in [3]) hypothesized that such phenomena accompanied by gamma radiation (a terrestrial gamma-ray flash - TGF) occur just before each ordinary lightning, that is much more often than it was thought. TGF-signal lasts about $70 \mu \mathrm{s}$ and is accompanied by a strong radio impulse. As the authors suggest, DL is not a phenomenon separate from ordinary lightning, but an intermediate stage of electrical discharges generation inside thunderclouds. Having lost some energy after their collision with air molecules and then giving rise to gamma rays, electrons provoke a powerful radio impulse followed by a visible electric discharge a in cloud, that is, ordinary lightning.

A mechanism for the formation of a chain of water quantum-coupled clusters, in which the rearrangement of hydrogen bonds (a structural phase transition) occurs synchronously, leading to an increase in the total voltage at the chain clusters is proposed in [3]. The coherent voltage causes the acceleration of electrons to $\mathrm{MeV}$ energies. This is the DL model where the acceleration mechanism is quite justified. Similar structures possibly occur in the atmosphere at moments prior to an earthquake, when both the lithosphere and the water-saturated cloud atmosphere are in quantum cohesion. Similar mechanisms should be involved into functioning of BL and other self-illuminating objects, such as sprites, jets, and even the lights of St. Elm, the lights of Hessdalen, often called as unidentified flying objects (UFOs).

\section{Model of BL in the form of three-dimensional skyrmion}

\subsection{Three-dimensional skyrmions are firstly observed}

Modern understanding of physical world along with its continuous technological development are based on classic electromagnetism and quantum mechanics. Quantum modelling of 
electromagnetic forces can inform about materials and systems which have no simple theoretical descriptions, such as these related to Hall physics or these which were not observed as, for example, magnetic anomalies. However quantum modeling which simultaneously realizes all main features of classical electromagnetism, has not yet succeeded. Interaction between a charged quantum particle and tied electromagnetic fields in a topological model of a ball lightning has been simulated experimentally. Induced by a precise space-time control of the spin field in the atomic Bose-Einstein condensate these phenomena are simultaneously creating the Shankar skirmion which is a topological excitation theoretically predicted four decades ago never observed before. The results revealed show universal possibilities of synthetic electromagnetism and give the first experimental images of topological threedimensional skyrmions in quantum systems.

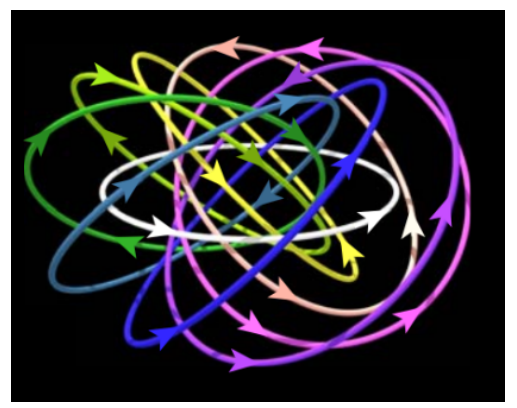

Figure 2. Skyrmion (named after its author Tony Skyrme) is a mathematical model in elementary particle physics used to modelling of baryons. Except baryons skyrmions are predicted to occur in the Bose-Einstein condensate, in superconductors.

Skyrmions as a special type of quasiparticles, which are vortex structures with reverse magnetization can occur in some magnetic materials. Nowadays, skyrmions have been obtained only in thin films on the surfaces of magnetic materials, and they all have a pronounced two-dimensional structure. However, theoretically it was predicted a possible existence of three-dimensional skyrmions, the so-called Shankar skyrmions, in which a special knotted structure of electric and magnetic fields occurs due to precise control of the spin field in Bose-Einstein condensate with the structure of oriented spins.

A group of physicists from the USA and Finland leaded by Wonjae Lee from Amherst College were the first to obtain three-dimensional skyrmions. They studied their stability and time evolution using a ferromagnetic spin-polarized Bose-Einstein condensate at a cloud of rubidium ${ }^{87} \mathrm{Rb}$ atoms, the state of spins in it can be governed changing the distribution of the external magnetic field.

Due to the linear combination of spatially distributed quadrupole and rapidly attenuating magnetic fields, the necessary spin structure in the condensate of atoms fixed in an optical trap was obtained. The orientation of the spins in this three-dimensional skyrmion varies continuously without discontinuities and singularities. When passing from one edge of skyrmion to the another the spin makes two full turns.

Physicists from Finland and the USA [1] have created and photographed an unusual quantum object, a kind of magnetic vortex, which is similar in its appearance and properties to BL. "It is very surprising that using two electric currents oppositely directed we were able to create a synthetic electromagnetic node, a quantum ball lightning (QBL). It suggests that natural BL of this type can arise from normal lightning strikes," - says Mikko Möttönen from Aalto University in Helsinki (Finland). 
$\mathrm{BL}$ is a ball of charged hot plasma. Appearing occasionally in atmosphere during thunderstorms it lasts longer than an ordinary lightning. It is unknown how they arise, and what processes control their movement and the researches argue about their nature for almost a century and a half.

Suggesting that BL are not only of electrical, but also the quantum nature Miettinen and his colleagues created a complete analogue of this "mystery of nature" in experimenting with the so-called skyrmions, the special objects of the quantum world.

Skyrmions are special hypothetical particles of matter with unusual magnetic properties that make them look like either a kind of "hedgehog" or a microscopic vortex. The tips of the needles in such a " hedgehog "or the edge of the hurricane are positively charged, and the body of the" animal" or the epicenter of the hurricane - is negative.

According to physicists, such quantum "hedgehogs" have an extremely high stability, which allows them to be used as long-term and economical memory cells in spin and quantum computers in future, as well as in ordinary computers now.

"Real" skyrmions are not yet created and their close analogues are special twodimensional structures inside thin films of magnetic materials such as manganese silicide where these vortices occur by themselves under special conditions, for example, at temperatures close to the absolute zero.

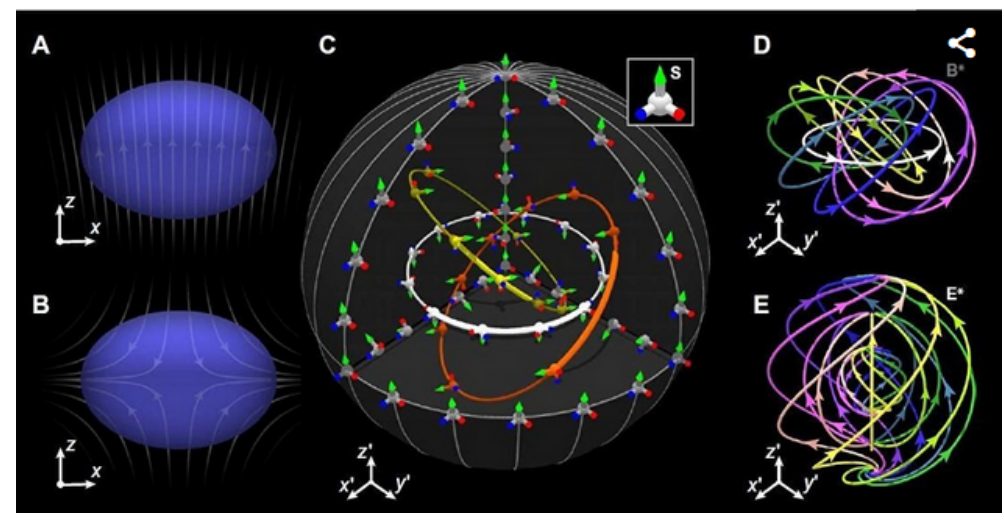

Figure 3. Creation of the Shankar skyrmion and its synthetic electromagnetic fields. (A and B) External magnetic field (white field lines) applied to the condensate (blue ellipsoid) just before (A) and after (B) the commencement of the creation process. The field has a continuous rotational symmetry about the $\mathrm{z}$ axis. The spin vectors are initially aligned with the magnetic field depicted in (A) but are reoriented as they precess about the new field lines (B) at their local Larmor frequency. (C) Cutaway octant of the created Shankar skyrmion texture exhibiting its continuous and topologically nontrivial triad texture. The inset shows a triad, for which the local spin direction $\mathrm{S}$ is marked by a green-tipped arrow. The blue- and red-tipped arms define the rotation angle of the triad about its spin vector. The colored curves are example contours, for which the spin vector points in a common direction, and along which the rotation angle winds about the direction of the spin by $4 \pi$. (D and E) Examples of the synthetic magnetic (D) and electric (E) field lines arising from the spin texture in (C), for which the colors are to guide the eye.

Using another quantum substance - the so-called Bose-Einstein condensate a new version of these particles with three - dimensional rather than two-dimensional nature was created by Finnish and American physicists. 
An unusual form of matter, similar to gas and liquid, it behaves like one giant atom and has typical "atomic" properties. It is obtained by cooling a cloud of rubidium atoms or other alkali metals to temperatures close to zero.

As Miettinen and his colleagues revealed, this artificial atom can be turned into a threedimensional analogue of skyrmion when put inside a strong magnetic field and the position of spin of each atom inside it is changed in a special manner. After this field is "switched off", a special quantum structure, which has the same configuration of electromagnetic fields braided into a node as the BL is generated inside the Bose-Einstein condensate.

As noted by the physicist the main unusual property of BL is that it exists a few hundred microseconds in a similar form that is an unusually long time for a quantum object suggesting according to Miettinen that a three-dimensional skyrmion is a "core" of BL, which also last an unusually long time compared to an ordinary lightning.

\subsection{Skyrmions are magnetic vortices together and separately}

Magnetic vortices of different physical nature: some exist individually, while others form a single structure were obtained in manganese silicide by Russian physicists.

Due to the own magnetic moment of the electron - spin, atoms in magnetic material behave like small magnets and, we can say, they themselves have a spin. When magnetizing, the spins of atoms are aligned in the same direction, turning the matter into a magnet. It was in 2009, when physicists revealed that during magnetization some magnets appear vortex structures of spins, in which spins of individual atoms as twisted in a certain direction (Fig. 4). These magnetic vortices have been called skyrmions, named after a British physicist Tony Scherma (Skyrme, 1922-1987), who proposed in 1962 a mathematical model of similar structure for elementary particles.
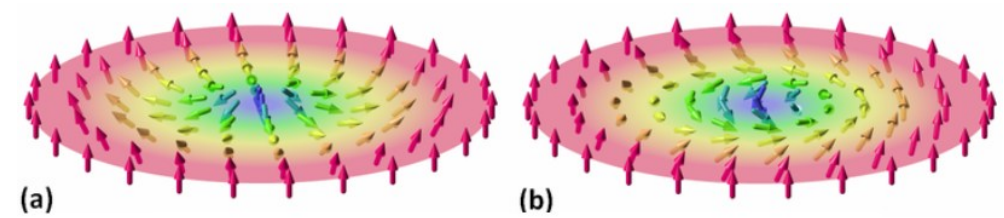

Figure 4. Skyrmions - two types of magnetic vortices: a) a hedgehog skyrmion and b) a spiral skyrmion. Arrows point to atom spins. (en.wikipedia.org).

Magnetic vortex structures have so-called topological stability owing to which during perturbations the twist is constant while the direction of the spins is changed. Believing an existence or absence of skyrmion for zero and one they can theoretically be used to store binary information due to their topological stability. Since the size of such vortices can be units of nanometers, it promises a significant increase in the density of information storage. Skyrmions might be applied to create logical devices. It would be possible to manage skyrmions with the help of an electric current.

Traditional magnetic domains (regions of the same magnetization direction) of such a small size are unable to store information for a long time due to their spontaneous remagnetization, which occurs under the influence of neighboring domains or due to fluctuations.

The properties of skyrmions are still poorly understood. Physicists from Russian MFTI and the A. M. Prokhorov Institute of General physics studied the behavior of skyrmions set in manganese silicide MnSi. The external magnetic field generates a structure of periodically located vortices in it, resembling bee cells with the size of about $18 \mathrm{~nm}\left(10^{-9} \mathrm{~m}\right)$. For 
the practical use of such a structure, it is necessary to know whether skyrmions exist independently of each other, or they form a whole structure, which is not separated into single vortices.

It turned out that the internal structure of matter can be determined if the resistance of the material depending on the temperature and the direction of the magnetic field is measured with a high accuracy. It was obtained in $\mathrm{MnSi}$ that at certain ranges of temperature and magnetic field there are two types of skyrmions lattice with a different physical nature, one of them is formed by the condensation of individual magnetic vortices, and the other corresponds to a complex magnetic phase, which cannot be decomposed into the individual skyrmions. There is a clear boundary between phases.

It is difficult to talk about prospects of this area of research, which is attributed to spintronics, because skyrmions are usually observed at very low temperatures. It is necessary above all to find materials that can best work at higher or at room temperatures. In addition, physicists are not yet able to create skyrmions reliably and manage them. According to the materials of MFTI https://www.nkj.ru/news/28477/

\section{BL simulated as dark lighting twisting into a ball}

\section{1 $\mathrm{BL}$ as a quantum phenomenon in atmosphere electric field}

BL is a natural phenomenon unique in many respects. First, it is far ahead of all others in the number of publications (ref. in [2]). Secondly, it is not still understood. Third, and most importantly, remaining in the frames of classical physics it is simply impossible to explain this phenomenon, otherwise, BL would be obtained in experiments with gas discharge. The only possible way to understand the nature of BL is to consider its physics using the achievements of quantum mechanics. The article [2] proposes a model in which BL properties are discussed in the context of non-local multiparticle quantum entanglement of protons in hydrogen bonds (HB) of water clusters exposed to lightning or other effects, for example, an electric discharge. Entering water clusters hydrogen bonds have the so-called cooperative properties meaning that all $\mathrm{HB}$, which are in a quantum entangled state, behave like a single cluster. Changing in one quantum state (for example, the destruction of $\mathrm{HB}$ ) instantly affects all others, entangled with it. The destruction of HB results in the appearance of unpaired electrons, their excess in atmosphere electric field leads to BL levitation, which provides BL with such observed features as listed below, they are its compactness, glow, acoustic and electromagnetic radiation, the ability to penetrate through micron holes, lasting at times from a few seconds to a minute, exploding, or fading away without an explosion, etc.

However, this model [2] like the BL model on skyrmion considered above, has no prerequisites to satisfy the criterion for model comparison chosen (Fig. 1). Otherwise, it is not clear whether BL holds its shape and stability after it is reduced to the size of the hole, i.e. when it practically wholly destroyed.

\subsection{Ordinary lightning problems}

The statement that BL occurs after the discharge of ordinary lightning is not correct. This condition is usually used in laboratory experiments to create BL. That is why in such experiments BL has never been obtained artificially. As in my own experience balls were observed, other than free-floating balls, lasting even for a minute, or even several seconds.

There are well-known BL without an ordinary one. BL even in an apartment with faulty wiring is known for me from my good friend and by literature. I studied the pinch effect in 
plasma to determine the possibility of BL formation. Pinch effect consists in plasma compression under the action of the current of about thousands of amps flowing through it. This phenomenon is observed in studying of electrical discharges with a high current in straight discharge tubes where surrounding itself with circular magnetic field lines the current interacts with its own magnetic field generating a force which compresses a discharge column into a narrow cord. After such a discharge, the remained electrons and ions recombine quickly. There are no quantum effects, water tetramers and dark lightning discussed here.

Pinch effect is peculiar to ordinary lightning. This effect is illustrated by a unique photo in Fig. 5-A.

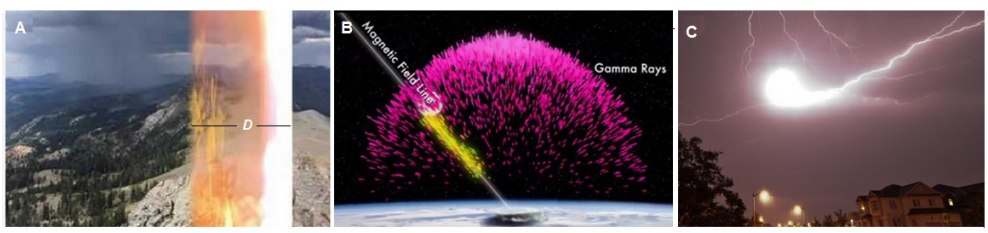

Figure 5. A - ordinary lightning (column of D diameter), a unique shot*, due to the pinch effect the lightning current is concentrated at the right side of the column; B - dark lightning observed by the Fermi telescope at NASA spacecraft. Red bursts - gamma- rays. Fast electrons (yellow) and positrons (green) propagate along the force line of the geomagnetic field. The signals were recorded at altitudes from $15 \mathrm{~km}$ up to $600 \mathrm{~km} \mathrm{[5];} \mathrm{C} \mathrm{-} \mathrm{BL} \mathrm{occurs} \mathrm{on} \mathrm{DL} \mathrm{[4].} \mathrm{*} \mathrm{Austrian} \mathrm{tourist} \mathrm{spent} \mathrm{his} \mathrm{vacations} \mathrm{in}$ the mountains of Northern California. After struck by lightning, he suffered severe burns, but he was able to move on his own. At the time of discharge, the tourist was taking pictures of the surrounding mountains. The resulting photo shows the vertical white-orange stripe which is the lightning. Earlier I have not seen such photos, although I am interested in the problem of lightning for more than 50 years. (www.province.ru/news/turist-sfotografiroval-na-mobi.html.)

Let's find out a question: if a thunderstorm cloud is able to provide the electric field necessary for the breakdown and the charges of required concentration [3]. The length of lightning recorded in 2007 in Oklahoma was 321 kilometers. In August 2012 super-lightning was sparkling in the sky over the South-East of France for as much as 7.74 seconds. It turns out that in most cases, the storm cloud cannot ensure the existence of strong lightning. Attraction of cosmic rays for additional ionization is possible, but it does not solve the problem. The scientific community solved the problem by attracting of DL, which precedes the ordinary one and creates the necessary concentration of charged particles. The model of DL appearance is proposed by the author [3].

\subsection{What is called by dark lightning}

Discovered in 1991 dark lightning (DL) is a stream of fast electrons moving at speeds close to light one, that sharply distinguishes it from ordinary lightning, which electrons are not so rapid. Colliding with air molecules ultrafast electrons of DL generate gamma rays, which give rise to electrons and their antiparticles, whose subsequent annihilation with air gives even more gamma rays. Nature arranges such a "performance" for a sole purpose - to provide a discharge of ordinary lightning.

In summer, 1991 at the conference on earthquakes prediction in Kamchatka, I reported about my shock-wave model of the earthquake [7]. One of the authors (ref. in [3]) asked me if I know a mechanism responsible for formation of $\mathrm{MeV}$-electrons flux at satellite altitudes directly in a seismically active environment shortly before an earthquake. Having no answer 
to this question a quarter of century ago I appear to be successful now after applying such a new concept of quantum mechanics as quantum entanglement.

\subsection{NASA experiment}

Recently, the media and scientific articles [5] informed about observations on NASA Spacecraft with the Fermi telescope, which was bearing atmosphere area above the storm. The authors believed that the source of gamma-ray bursts (TGFs) were strong electric fields concentrated at the top of thunderclouds. Gamma-ray burst (TGF) generates gamma-ray radiation (red), as well as fast electrons (yellow) and positrons (green). This model traces the trajectory of TGF and the particle beams generated by the burst from a sea level to the height of $15 \mathrm{~km}$ up and further up to $600 \mathrm{~km}$ which is outside the orbit (Fig. 5-B). This figure shows that the phenomenon under consideration is clearly related to the magnetic field line, as it follows from our model (Fig. 6). And that is all we know about DL. There is no answer to the question of how DL is formed.

\subsection{Dark lightning, its model}

In [3] we propose a mechanism for the emergence of a quantum-coupled water clusters chain, where hydrogen bonds simultaneously (coherently) restructure (a structural phase transition) followed by an increase in the total voltage at the chain clusters, which are water tetramers.

Offering a potential and a dipole moment the tetramers line up along the magnetic field line (Fig. 6-1) so that the signs of the charges alternate and the total potential of the electric field along the line increases. The resulting coherent voltage causes the acceleration of electrons up to MeV- energies. This is the DL model, and the mechanism of electron acceleration is sufficiently justified by its use in accelerators. Similar structures are possible to occur in atmosphere at moments preceding an earthquake, when both lithosphere and water-saturated atmosphere are in quantum entanglement. Similar mechanisms may be involved in the functioning of other self-illuminating formations such as sprites, jets, St. Elmo's and Hessdalen's lights, often referred to as unidentified flying objects (UFOs).

\subsection{Water tetramers}

Water tetramers $\mathrm{H}_{8} \mathrm{O}_{4}[3,6,7]$ are the most stable water clusters. Tetramer is a triangular pyramid in which two vertices have positive charges due to the proximity of protons and the other - two negative charges due to the influence of oxygen atoms. Each tetramer has four hydrogen bonds, denoted as $(\mathrm{O}-\mathrm{H})$, the equipotential surface of tetramer with unevenly distributed charges is quadrupole and can be characterized by a potential $\varphi$. These tetramers line up along the magnetic force line (Fig. 6-1). It is important for our DL model: if there is a fundamental restriction of the associates number in the chain, or, all changes in water structure occur in the range from the tetramer $\mathrm{H}_{8} \mathrm{O}_{4}$ to oligomers $\left[\mathrm{H}_{8} \mathrm{O}_{4}\right] n$ and their spatial association.

The electric field strength $E$ is related to the potential $\varphi$ as $E=-\operatorname{grad}(\varphi)$.

The deformation of the equipotential surface of the quadrupole, leading to a change in its size, causes a change in the potential: $\Delta \varphi=q / 4 \pi \varepsilon_{0}\left(1 / r_{1}-1 / r_{2}\right.$. Here $r_{1}$ and $r_{2}$ - the size of tetramer before (A) and after (B) its deformation (Fig. 6-1). Thus, deforming the structure of hydrogen bonds in tetramer, it is possible to obtain an additional potential $\varphi$.

Tetramers are so stable that they do not break down even at water boiling point $\left(100^{\circ} \mathrm{C}\right)$. From IR spectroscopy data, their partial destruction starts at $200^{\circ} \mathrm{C}$ with a complete destruction of $\mathrm{H}_{2} \mathrm{O}$-monomolecules to atoms at temperatures above $2000^{\circ} \mathrm{C}$. 


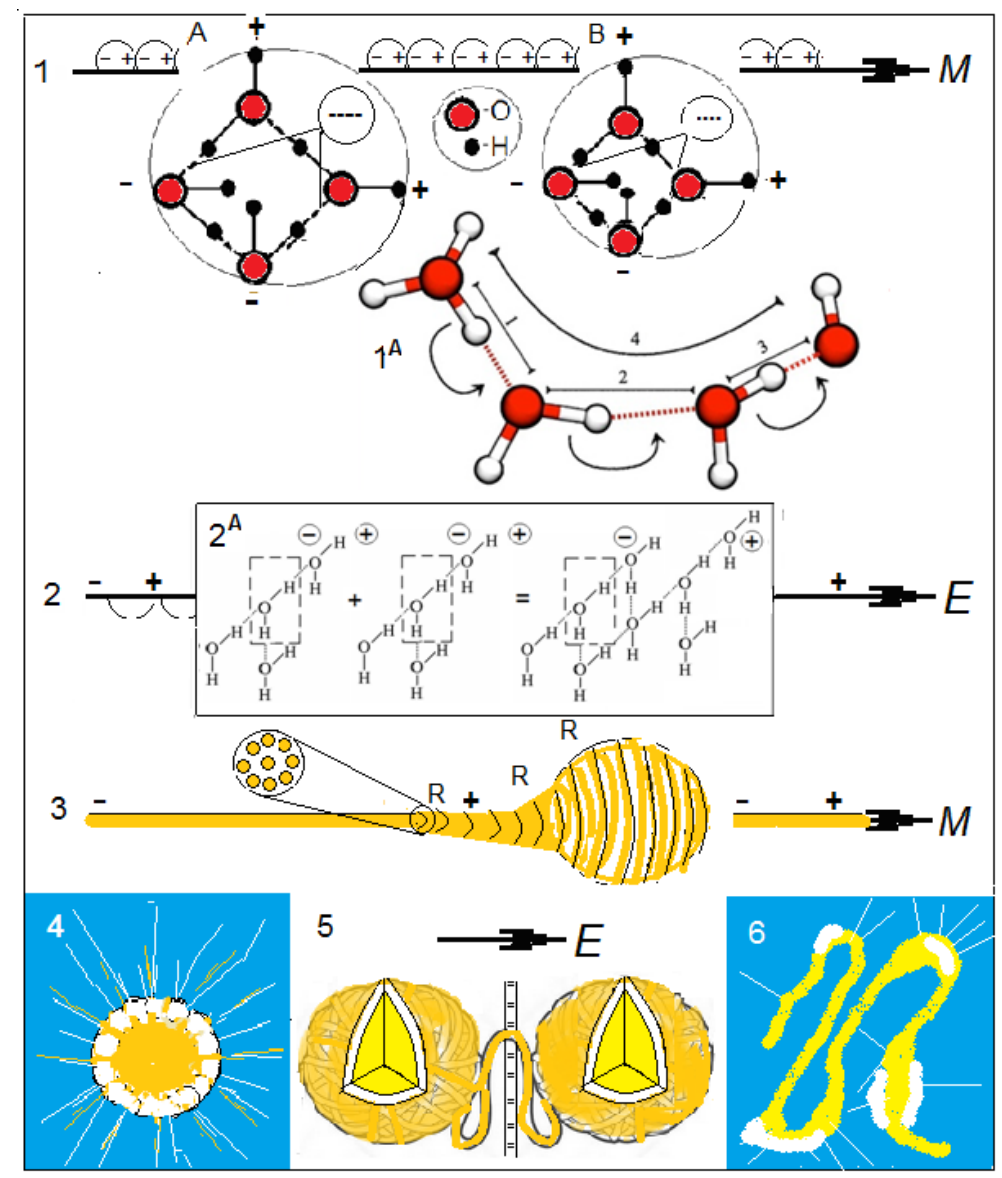

Figure 6. Model of BL: 1 - Clusters - water tetramers $\mathrm{H}_{8} \mathrm{O}_{4}$. A - tetramer with usual hydrogen $(\mathrm{O}$ $-\mathrm{H}$ ) bonds $(---), \mathrm{B}$ is the same tetramer with shortened $\mathrm{OH}$-bonds (????), $\mathrm{M}$ is a force line of the geomagnetic field $\mathrm{B}, \mathrm{1}^{\mathrm{A}}$ - is the contact pair of ions $\left(\mathrm{H}_{3} \mathrm{O}^{+}, \mathrm{OH}^{-}\right)$through which protons are transported (in arrows direction). 2 - summing the electric fields of tetramers provide the field $\mathrm{E}, 2^{A}-n$ tetramers $\left(\mathrm{H}_{8} \mathrm{O}_{4}\right)$ add up: $\left(\mathrm{H}_{8} \mathrm{O}_{4}\right) \cdot n$, here $n=2$ [6]; 3 - the yellow line consisting of parallel chains of tetramers is a DL, which transforms into BL at its breaking under the magnetic field B effect; 4 - BL, directions of $\mathrm{MeV}$-electrons drift are shown; 5 - BL knot approaches glass with a tiny hole, where the end of the cord enters (the hole size should be not less than the thickness of the cord); 6 - a piece of DL cord broken is a slightly luminous formation, taken as a UFO.

Energy up to $40-50 \mathrm{~kJ} / \mathrm{mol}$ per each H-bond yields $120-150 \mathrm{~kJ}$ for tetramer. This large value of $\mathrm{H}$-bond energy in the associate allows to consider the tetramer $\mathrm{H}_{8} \mathrm{O}_{4}$ as a separate single molecule [6].

Co-operability is a well-studied property of water, when its molecules oscillate synchronously under the action of some force and therewith the structure of hydrogen bonds may change (they "bend" or "shorten" or tear through a structural phase transition (SFT) when some energy is released (FT-heat). Here the equipotential surface of the tetramer changes and an uncompensated electric charge $q$ may occur. This charge $q$ is very small. Broadly speaking, the value of $q$ should not be greater than the unit charge of the electron $\left(1.6 \times 10^{-19}\right.$ Coulomb). For a rough estimate, we take $q=10^{-20} \mathrm{~K}$. Assume that the surface 
is changed by $\Delta r \approx 1$ Angstrom $(\AA)=10^{-8} \mathrm{~cm}$ and the size of the tetramer is $R_{T} \approx 10 \AA$. We can estimate the value of the field $E$ for the tetramer size: $E=q / 4 \pi \varepsilon_{0} R r \approx 10^{9} \mathrm{~V} / \mathrm{m}$ $(0.1 \mathrm{~V} / \AA$ A $)$ and the potential of one tetramer $\varphi=1 \mathrm{~V}$.

If $10^{7}$ tetramers are located at one centimeter then the voltage $U$ is of the order of $10^{7} \mathrm{~V}$ which provides a possibility of not only x-ray radiation, but also of electrons acceleration to high $\mathrm{MeV}$ values. The probability of such electrons is negligible, but not zero. In plasma physics, such electrons are called "escaping" ones [8], they are electrons escaping from ions.

The chain of ions, shown in Figure 6- $1^{A}$, is involved into a transfer of protons which enter the area of growing BL where under the action of the earth's magnetic field B they begin to move along the R radius with the velocity $V: V=q B R / m$. Here $q$ is the charge of proton, $m$ is its mass. The velocity of the proton drift with tetramers incorporated is a thermal velocity $V=\sqrt{3 k T / m}$. Through the elastic collisions protons provide the rotation of tetramers in DL piece from which BL appears (Fig. 6-3). Tetramers, i.e. actually, water are heated in so doing, it leads to a further increase in the flow rate $V$ and even to greater heating of water in BL. Here a part of water turns into vapour, which provides BL "volatility". Thus, being of great interest in technical physics in recent years a steam generator mode is implemented in BL. It turns out that Nature, in particularly the nature BL, has already used this effect.

\subsection{The occurrence of $B L$}

Let's take the radius of BL equal to $7 \mathrm{~cm}$ (Fig. 7-3) and the ball volume $V \approx 1500 \mathrm{~cm}^{3}$. After the destruction of DL, tetrameters turn into chains of quantum-coupled water molecules located along the magnetic field line in the form of charged dipoles. Rotating in a plane transverse to the axis of the magnetic field the chains gather into a knot (Fig. 5-C, 6-3). Since the elements of these chains are almost unbending edges of tetramers and the angles of tetrameters are strictly specified, the initial radius of the knot cannot be arbitrary small. Thus, the resulting knot will probably be hollow. This model satisfies Fig.7-3, which shows the well-known distribution of matter density along the radius of BL. The density of BL matter in the knot center is less than the air density. Common for BL Fig. 7-2 corresponds to our model of BL formation (Fig. 6-4).

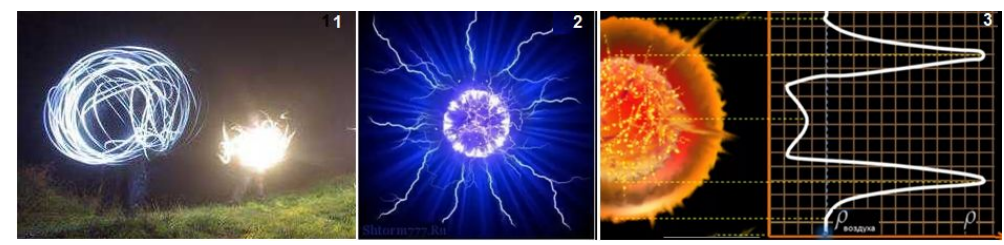

Figure 7. 1 - Stages of BL formation [4], 2 - photo of BL, 3 - distribution of BL density by its radius (http://fireboll-new.ucoz.ru/index/plotnost_veshhestva_sharovoj_molnii/0-47)

BL movement in atmosphere (its volatility) is based on Archimedes' law. If BL gravity $m g$ together with that of water vapour filling the shell is less than the weight of air in the volume displaced by BL shell, the ball rises; if they are equal, the ball hangs motionless in air; if the gravity $m g$ is more than air weight, the ball falls.

From numerous observations of BL behavior, it occurs immediately, with virtually no precursors. According to our model, BL arises from DL, and the time iterval between them is not small. This consideration is seemingly contradictive to our model, but in Internet there is a picture (Fig. 5-C) which shows that BL is developing at the rupture of DL for a time, sufficient at least to make a shot. Moreover, it is fundamentally important, that both BL and 
DL coexist for some time and already glowing BL releases heat which heat air. "Extra "air flows out providing in BL "volatility".

\subsection{Processes inside BL}

Tetramers are to be located at the outer wall of BL (Fig. 7-2). The unit weight of BL is to be equal to that of air $\varrho_{B L}=\varrho_{a}$. All phenomena inside BL are provided by tetramers presence and their number. Here are the most basic estimates. Let us take the tetramers weight equal to $2 \mathrm{~g}$. This value is chosen from the assumption that this is the minimum weight of water in BL. Similar estimates can be made for more large weight. The number of tetramers and their layer thickness in BL $\delta: \delta=V_{t} / 2 \pi R_{B L}^{2}=2 \mathrm{~cm}^{3} / 300 \mathrm{~cm}^{2}=60 \mu \mathrm{m},\left(V_{t}\right.$ a volume of tetrameter). The number $N=V_{t} / R_{t}^{3}=2 \mathrm{~cm}^{3} / 10^{-21} \mathrm{~cm}^{3}=2 \times 10^{21}$.

Assuming that at the time of the phase transition the energy of about $1 \mathrm{eV}$ is released at each tetramer, the total electrical energy of all tetramers is $E=2 \times 10^{21} \mathrm{eV}$, or $\approx 300 \mathrm{~J}$. If the destruction energy of one hydrogen bond is about $400 \mathrm{~kJ} / \mathrm{mol}$, and one mole of water $\approx$ $20 \mathrm{~g}$, we obtain the mechanical energy of BL equal to $20 \mathrm{~kJ} / \mathrm{g}$, that is 5 times higher than the energy of TNT $(4 \mathrm{~kJ} / \mathrm{g})$. At the tetramers mass of $2 \mathrm{~g}$, the total mechanical energy of their complete destruction is significantly (almost 100 times) higher than their electric energy. Thus, if BL explodes with synchronous destruction of all its hydrogen bonds, such effect is comparable to the explosion of a hand grenade. This comparison is not entirely legitimate because the initiation of TNT explosion requires a detonator. To destroy simultaneously the hydrogen bonds in all tetramers, it requires all their hydrogen bonds to be in a state of quantum entanglement $(\mathrm{QE})$, but we do not know how to create $\mathrm{QE}$, or manage it. To be honest we do not understand its essence. Nevertheless, the use of this approach allowed us to create a so-called the shock-wave model of an earthquake, here the hydrogen bonds of lithosphere synchronously change in such a way that a coherent flow of acoustic pulses, which is converted into a shock wave (SW) occur. Reaches lithosphere surface SW holds a maximum possible energy that is near to that of the hydrogen bomb. Thus, the comparison of BL energy with the energy of a hand grenade is not an author's overstating.

\subsection{BL as a chain of tetramers twisted into a ball}

Fig. 6 shows a sequence of tetramers chain located along the magnetic force line (Fig. 6-1) and their total electric field E increases (Fig. 6-2). Representing a basis of DL the chain twist, forming in a magnetic field $M$ a knot which transfotms into BL (Fig. 6-3). The brightness of the expanding DL with the knot increases (Fig. 5-2), that suggest processes inside BL such as formation and acceleration of electrons, ionization and excitation, elastic collisions, resulting in heating of tetramers and air captured, which expands and leaves BL providing its volatility required. Arising in tetramers the electric charges can now be added not along the chain, but along the radius of $\mathrm{BL}$, from layer to layer, and, accordingly, the acceleration of electrons occurs by the knot radius. Fig. 6-4 shows the glowing tracks of fast electrons.

At Fig. 6-5 BL penetrates a very small gap. Untwisting at one knot the DL cord is twisting at another. Losing the influence of the magnetic field the torn off piece of BL does not twist into the knot remaining in a "crumpled" shape (Fig. 6-6) which is called "Hessdalen lights" observed often after lightning strikes.

Let's take the initial data for further assessments. The energy of BL assessed falls into its range of volumetric energy from 2 to $100 \mathrm{~J} / \mathrm{cm}^{3}$. The average radius of BL observed to be $\approx$ $7 \mathrm{~cm}$ and its volume $\approx 1500 \mathrm{~cm}^{3}$ correspond to the minimum estimate of BL energy $\approx 5 \mathrm{~kJ}$.

Shining and heating like a light bulb BL goes out with its hissing after 100-1000 seconds. The energy of DL electrons is about $1 \mathrm{MeV}$. Suppose that these electrons are decelerated by 
air. As a result, we observe a glowing track of their decelerating as seen in Fig. 7-2. A part of the electron energy is used for air ionization and appearing electrons are accelerated by the tetramers field. This electric field is attenuating after the generation cycle when HB varies in size. The cycles repeat until HB is capable of size changes or discontinuities. This mechanism is of the same nature as at aftershocks following an earthquake. As we noted above, the collective rupture of $\mathrm{HB}$ generates BL explosion.

As noted above, the heating of water tetramers occurs mainly due to their rotation transmitted by protons rotating in a magnetic field, i.e. due to a thermal generator in BL. Heating results in an increase of water velocity $\mathrm{V}$ and the ball radius $\mathrm{R}$. Maximum temperature of tetramers destruction is more than $2000{ }^{\circ} \mathrm{C}$. This value is very important for BL model, because BL matter is taken to be a hot plasma. BL temperature was repeatedly recorded to be very low. A few words about BL hissing. A similar phenomenon is known to anybody who was walking near the melting ice in the spring sunny day. The sound is associated with the destruction of HB. Similar phenomena involve the so-called "a song of water", sounds in oceans and atmosphere. (The author talked about these sounds in the film "The Noise of the Earth", http://www.vvkuz.ru).

\section{Conclusions}

Two models of BL are discussed. The model of skyrmion is obvious not to satisfy the criterion chosen. There are some other parameters that are not suitable for real BL, for example, the fact that this model is two-dimensional operating at zero temperatures, etc.

The model of BL as a twisting knot proposed here is also not free from criticism. The fact often observed is that BL appears fastly and it has no precursors. However, other BL parameters, such as its sound (hiss), slow dimming, BL explosion follow from our model. It is known that the transformation of HB lengths is associated with the generation of acoustic impulses.

The second model proposes a mechanism for the emergence of a chain of water quantumcoupled clusters, in which hydrogen bonds are simultaneously and coherently restructuring (a structural phase transition), that leads to an increase of the total voltage at the clusters of the chain. The coherent voltage causes the acceleration of electrons up to $\mathrm{MeV}$ energies. This is a model of DL, and the mechanism of acceleration is quite justified. It is quite possible that similar structures arise in atmosphere at moments preceding an earthquake, when both lithosphere and water-saturated atmosphere are in quantum entanglement. Similar mechanisms may be involved in activity of BL and other self-illuminating objects such as sprites, jets, and the lights of St. Elm and the lights of the Hessdalen valley, often referred to as unidentified flying objects (UFOs).

Water taking part in BL generation in the form of a thin layer of tetramers is transparent, that is often surprising to BL observers. Ordinary lightning is mostly transparent too (Fig. 5-A). Water involved into BL formation is quantum entangled. It is usually rain water. This should be considered in experiments to create a laboratory sample of BL. We offer an experiment based on our model:

First, you should understand how to create DL sample. You need a container filled with rain water and a long thin glass tube with a coil producing an axial magnetic field inserted into the container. Tetramers in a magnetic field will link to each other forming a long chain. After "running" electrons start their moving along the tube, DL is ready. If it doesn't happen, then it is necessary to extend the tube, or to apply a static electric field to the tube.

Secondly, BL should appear at the open end of the tube. The ball should grow to a certain size, determined by the magnitude of the magnetic field at the tube, then it should tear off and float in air. It means that BL is created! In the case of failure - our model is wrong. 


\section{References}

[1] Lee W., Gheorghe A.H., Tiurev K., et al. 4, DOI:10.1126/sciadv.aao3820 (2018)

[2] Kuznetsov V.V., Kvantovaya magiya 8, 3101-3107 (2011), in Russian

[3] Kuznetsov V.V., Vestnik KRAUNC. Phys.-Mat. Science 4(15), 63-73 (2016). DOI:10.18454/2079-6641-2016-15-4-63-73, in Russian

[4] Galanin A.V., Universe is alive (Vladivostok, 2013) http://ukhtoma.ru/dinamic9.htm, in Russian

[5] Dwyer J.R., Geoph. Res. Lett. 117, A02308 (2012)

[6] Nikolaev A.F., News SPBSTI (TU) 1(27), 110-115 (2007), in Russian

[7] Ignatov I., Mosin O.V., Velikov B., The Institute of Public administration, law and innovative technologies (HOPIT) Internet-journal "science of SCIENCE" 3, (2013) http://publ.naukovedenie.ru

[8] Gurevich A. V., Zybin K. P, Physics-Uspekhi 171(11), 1177-1199 (2001), DOI: https://doi.org/10.3367/UFNr.0171.200111b 